\title{
Physiological Aspects of Physical Measurement.
}

By Sir John Herbert Parsons, C.B.E., F.R.S.

PHysicists too often forget that the basis of 1 physical measurements is biological, for the sothe sensations it arouses in our bodies. Physical measurements are open to the errors of all human observations, and these vary in degree according to the type of observation. In all cases the observation is the formation of a judgment, based on the sensations derived from the stimulation of a sensory organ. Physiological experiments show that stimulation of some sensory organs gives more sharply defined responses than others. Thus, the responses to smell and taste are crude and vague; those to moderate cutaneous stimuli-touch and temperature -much better defined; those to auditory stimuli, still better, and those to visual best of all.

But even among the varieties of a given type of sensation various degrees of definition are met. Thus pain, though cutaneous, is crude like smell and taste in vision, form sense is much more accurately defined than colour sense. Definition, indeed, varies as the biological differentiation of the sense organ.

Now, the most highly differentiated sensory organ is the eye, and the fovea is its most highly differentiated part. Experiments show that the greatest discrimination is met with in foveal stimuli. The highest degree of sensory discriminatior is the appreciation of continuity or lack of exact continuity in two straight lines set end to end, as in the vernier. This may be called linear identity, and it is noteworthy that it has been adopted empirically by physicists in the vernier, balance, and other instruments. Physicists have been very ingenious in applying this criterion to otherwise apparently unsuitable measurements, as, for example, the measurement of temperature. 'But there are many physical measurements to which it cannot be applied, or at any rate has not been applied. Photometry is an example. Here we are measuring the brightness of two lights. By various devices the principle of identity or equality of sensations is made use of-thus utilising the only accurate psychological comparison-but the quality of the sensation to be adjudicated upon does not admit of the accuracy of linear identity. Even in homochromatic photometry we are comparing the brightnesses of two illuminated areas. As is well known, these areas react upon each other physiologically - by the process of induction or simultaneous contrast. Moreover, the judgment is affected by the previous stimulation of the retinal areas concerned (successive contrast) It is further vitiated by variations in adaptation.

Still more open to error are the comparisons of brightness of different coloured lights, heterochromatic photometry. Here the difference in colour acts as a very disturbing element. 'Yet by practice it is possible to attain almost as accurate results as in homochromatic photometry. But how can we judge of the accuracy of these determinations? In this particular instance we can have recourse to the fact that the critical frequency of flicker depends upon brightness and follows a definite mathematical law. The eye is extremely sensitive to flicker, so that the disappearance of flicker affords a very sensitive criterion. It has been found that the results obtained by the flicker photometer confirm the results obtained by the best so-called "equality of brightness" observations.

No matter how delicate the criterion there are still errors of observation due to imperfections of a bio1 From the presidential address to the Illuminating Engineering Society,
delivered on May 25. logical nature common to all human observers and also to the so-called "personal equation" of the given observer. How are these to be eliminated? Recourse is had to mathematical theory. The basis of the theory of error, which is a branch of the theory of probability, is that small errors will be more frequent than large ones, very large ones will be practically absent, and the mean is the result of the mutual destruction or compensation of many small sources of error acting in opposite directions.

The kinetic theory of gases is built entirely upon this statistical foundation, and its success in explaining the physical properties of gases is strong evidence in favour of the statistical theory. There are several mathematical " averages or means," and much depends upon the choice of the suitable "means," which itself depends upon the frequency distribution of the observations. Graphic methods of eliminating errors are constantly used by physicists. One of the commonest is the method of interpolation, and the smoothing of the curves.

An interesting example of the opposite aspect of averages is the modern view of atomic weights. These are some of the most accurate physical measurements ever made and have been corrected by the best statistical methods. Many of them approximate nearly to whole numbers and there are many theoretical reasons for believing that they are whole numbers. Recent investigations, chiefly by Aston, have shown that the atomic weights hitherto obtained are themselves averages: that there are many socalled " isotopes," having almost if not quite identical chemical properties, but differing from each other in the number of their electrons and also in their true atomic weights, which are invariably integers.

I hope that this philosophical parenthesis suffices to show that even in the matter of physical measurements the physiological aspects of the subject must perforce be taken into account. But in dealing with illumination we are dealing not only with foveal vision, but also with peripheral vision and alterations of sensitiveness of the eye under different conditions of stimulation. It is well known that the foveal region of all parts of the field of vision alters least in sensitiveness under different intensities of illumination. It is, therefore, relatively stable, and observations founded on criteria derived from central vision are proportionately trustworthy. It is quite otherwise with the other parts of the field of vision. Here the sensitiveness of the retina increases enormously with diminution of the intensity of stimulation. This function of retinal adaptation, which is of such tremendous practical importance in the life of the individual and indeed of the species, interferes very seriously with the accuracy of scientific investigations. Physicists have been led astray by ignoring it, as, for example, in the so-called "deviations from Newton's law of colour mixtures" described by König.

Physicists, indeed, are so accustomed to deal with measurements of the highest order of accuracy, founded upon what I have called "linear identity", observations, that they succumb to two errors: (I) that of regarding these observations as of the supreme validity of mathematical abstractions; (2) that of regarding other observations, to which the "linear identity" criterion is inapplicable, as of far greater accuracy than is in fact the case. When the mistakes arising from these errors are too patent to be ignored, physicists are apt to exhibit an unwarranted impatience with the shifting sands of

NO. 2772 , VOL. Y IO] 
biological science. The fact must, however, be faced that in all cases the observing instrument is a living organ and is, therefore, in a perpetual state of change. The rate of change is relatively slight in the most favourable cases, but rapid and complex in the less favourable. Physicists have been notoriously successful in so reducing the physical complications of experiments to a minimum that the problem rearly approximates to a mathematical abstraction, and, therefore, the highest degree of accuracy. Further advance is to be sought by greater attention to the biological complexities in order that they, too, may be subject to more complete control.

A mass of evidence has of recent years accumulated to show that in peripheral vision two mechanisms are simultaneously at work. Of these, one is chiefly concerned with vision under low intensities of lightwhat I have called scotopic vision. The end organ of this mechanism is the rods of the retinal neuroepithelium. Photopic vision, or what may be called daylight vision, is chiefly carried out by the cones. The duplicity theory is so well established that it has even found its way into the writings of the physicists. The explanation and our knowledge of retinal adaptation depends upon these physiological facts. Since retinal adaptation plays a preponderant part in simultaneous and successive contrast its importance in photometry will be readily realised.

\section{The Design of Railway Bridges.}

\begin{abstract}
$\triangle$ SUBJECT of great importance to the general public is the safety of the thousands of bridges by means of which our railways cross roads, rivers, and other railways. Probably it occurs to few railway travellers to consider the complexity of the design of each bridge they cross and the organisation required to inspect, test, and maintain every bridge in a condition suited not only to the traffic for which it was originally designed, but also to the increased weights and speeds which have since been introduced. It is but natural that differences of opinion should arise between the railway companies which have to pay for their erection and maintenance, and the Board of Trade which has to satisfy itself that they are safe.
\end{abstract}

The Ministry of Transport has recently carried out a series of tests on actual bridges, and has issued a report containing suggestions which appear to foreshadow regulations requiring railway bridges to be heavier and therefore more expensive. This report has naturally aroused great interest and caused no small concern among the bridge engineers of the leading railway companies. At the meeting of the British Association at Hull, the Engineering Section devoted a morning to a discussion of the problem. Unfortunately no representative of the Ministry of Transport took part in the discussion, but the railway companies were well represented and the speakers included the bridge engineers of the Great Western, North Eastern, and Great Central companies. Taken together the papers constitute a concise but fairly complete review of the present situation.

Mr. J. S. Wilson, who opened the discussion with a general review of the questions involved, showed that the difference of opinion between the companies and the Board of Trade is nearly as old as the railways themselves. In 1849 Torksey Bridge across the Trent was tested by the representative of the railway commissioners preparatory to the opening of the Retford and Lincoln line. The deflection of $\mathrm{I}+$ inches with four locomotives and tenders on the centre of a span was considered excessive and permission to open the line was refused. The bridge had been designed by John Fowler who, with Sir Benjamin Baker, was responsible later for the Forth Bridge. He had followed the rules laid down by Fairbairn, and he suggested to the commissioners that some mistake had been made, but after further tests the latter persisted in their view that the stresses in the bridge were excessive. Finally, however, Fowler succeeded in convincing the commissioners that the girders, being continuous over the middle pier, were not stressed so highly as would otherwise be the case, and on his offering to reduce the weight of ballast on the bridge, the line was finally opened after a delay of three or four months.
The subsequent history of the bridge is of interest. The bridge is still there; for forty-six years it was unaltered and carried all traffic satisfactorily; in 1896 it was strengthened by the addition of a longitudinal girder. These old iron bridges designed by Fairbairn and Fowler, which have stood the test of seventy years' wear and tear and are still in good condition, are powerful arguments in favour of the view that bridges built on the same assumptions will be perfectly safe.

There are many difficulties, however, in the calculations and assumptions involved in the design, in allowing for the effect of impact due to the fact that the load is a live one, that is, not a stationary load, and in allowing for the effect of sleepers, rails, and ballast in strengthening the structure, distributing the load, and damping out the effects of impact. It is here that some doubt arises as to whether the intentions of the Ministry are correctly interpreted by the railway companies; it is useless to specify a factor of safety or a working stress unless one also specifies how the stress or factor is to be calculated or determined. The bridge designer may employ what appears to be a low factor of safety because he knows that the actual stresses are less than those calculated by the simple conventional methods usually adopted and that his actual factor of safety is consequently much greater. If a high factor of safety is specified, then it is open to the engineer to modify not his design but his methods of calculation so as to take account of the various strengthening factors usually neglected, and thus obtain a lower calculated stress and a higher factor of safety than would be given by the usual semi-empirical method.

One speaker in the discussion advocated making full-scale tests on old bridges which were being replaced; these could be re-erected and thoroughly tested, if necessary to destruction. All the speakers deprecated the premature promulgation of rules which would lead to heavier and therefore more expensive bridges, but urged that present practice should be followed until systematic research has been carried out and far more knowledge of the subject obtained than that on which the Ministry of Transport are proposing to act.

In their attitude towards riveted structures of iron and steel, engineers may be divided into pessimists and optimists, and Mr. Wilson's experience showed that the greatest optimists have been those most closely associated with the maintenance or actual construction, who would certainly be the first to detect any indication that the bridges were showing signs of weakness.

An engineering student is always taught that the stress produced by a live load is double that produced by the same load when steadily applied. This 